\title{
Multi-Senor QR Decomposition of the Matrix Fusion Interval Kalman Smoother
}

\author{
Guobo Xie, Long Guo, Wen Lu \\ School of computer ,Guangdong University of technology ,Guang Zhou, China \\ E-mail: guoboxie@163.com
}

\begin{abstract}
In this paper, a new algorithm is proposed for computing weights fusion of fixed-interval Kalman smoothers in the linear minimum variance sense. The QR decomposition of matrix is presented for the weights for discrete time-varying linear stochastic control systems with multiple sensors and correlated noises. Theoretical predictions and numerical evidences indicate that the algorithm of QR decomposition of matrix to value the weights fusion fixed-interval Kalman smoothers can save dramatically the memory and alleviate considerably the computational burden, but don't loss any numerical precision.
\end{abstract}

Keywords - fixed-interval Kalman smoothers ; the QR decomposition of matrix ; multiple sensors ${ }^{1}$

\section{Introduction}

With the development of the times, especially in the 21st century,Modern communication technology and microelectronics technology and so has been rapidly, developed, Specifically in terms of military, economics use multi-sensor to these complex background environment, so that people are able to get more than single-sensor data information to facilitate the extraction and analysis, the people to make the analysis more accurate, machine running the more stable. However, The more sensor is the more data people to deal with and makes people have to make a more and new in-depth study of multi-sensor information processing, in order to obtain more effective information processing for current and future. Although It has become popular areas for people to study, it has not yet formed a mature scientific system, its development began in the military field, track positioning, now, more and more use of things such as the rise of the multi-sensor industry to the lives of ordinary people, so far, the development of the industry, has made a good start, Wiener [1] put forward Wiener filtering method of filtering noise from the contaminated signal in the frequency domain, and reached the real optimal estimation of the signal, in the linear minimum variance sense both filters. The disadvantage is that require steady signal and store all historical data to calculate engineering use, this method of computation and storage are too enormous to promote. Carlson [2] presents the famous federated square root filter. That use the upper bound of the process noise covariance matrix to replace the process noise covariance matrix, also considered the estimation error correlation between subsystems, given fusion filtering algorithms in the linear minimum variance sense ,But, to some extent, it is conservative because of using the upper bound of

\footnotetext{
${ }^{1}$ This research is partially supported by the Combination of the Ministry of education project in Guangdong Province.
}

the variance matrix of the process noise instead of the variance matrix itself. Kalman [3] raise filtering kalman algorithm in the time domain, it use Riccati equation to establish equation of state , using recursive filtering algorithm does not require a lot of storage, conform to the development of the electronic computer, military and space technology to overcome the shortcomings of the wiener filtering method has been widely used. Literature [4] gives a fusion criterion weighted by scalars for systems with multiple sensors. But the assumption for the state estimation errors between any two uncorrelated sensors doesn't accord with the general case. So [4] only get a sub-optimal information fusion filter. Distributed fusion filter requires each subsystem with the same dimension of the observation matrix in the [5].This makes it has limitations in the application, In practical applications, due to the system model is unknown, and maybe change with time-varying, Also, because of the different types and the observation position of the respective sensors, the system model describing the target of the respective sensors may also be different, Thus, either a single model of the system is sometimes difficult to describe the state of the actual motion of the system. Kim [6]and Chen et al. [7] give the optimal fusion filter for systems with multiple sensors based on the maximum likelihood estimation, respectively, and assume the process noise to be independent of the measurement noises.But its derivation requirements .Assuming the estimated error is based on normal distribution. Weighting by matrices need to calculate the inverse matrix, the large amount of calculation .

This article use the QR decomposition method, the matrix inverse calculation problem is solved to some extent, promote the weighted matrix algorithm optimization. Distributed multi-sensor information fusion has high reliability, viability and short decision-making time and cause of widespread concern, and has been extensively studied. Kalman filter is mainly used for the integration of low-level real-time dynamic multi-sensor redundant data. This method is recursive, the statistical properties of the measurement model to determine statistical significance optimal integration and data estimated. If the system has a linear dynamic model and system sensor error Gaussian white noise model, the Kalman filter for the fusion of data provide only statistical significance optimal estimation. Kalman filter recursive features make the system does not require a lot of data storage and computing. However, a single Kalman filter statistics on the combination of multi-sensor system, there are many serious problems EKF'S advantages: the error of the linear extent of the 
instability of data processing or system model can effectively overcome the fusion process the impact. the overall physical laws better understood.

\section{Research method}

In this paper, three distributed optimal weighted fusion fixed-interval Kalman smoothers with a three-layer fusion structure are given for discrete time-varying linear stochastic control systems with multiple sensors and correlated noises based on three optimal matrices weighted fusion algorithms in the linear minimum variance sense. this will be smaller computational higher accuracy than direct matrix weight, The third chapter is divided into three parts, the first part: the basic formula assumes the second part of the matrix weighting method QR the matrix de-composition wears fusion, the third part of the simulation comparison. The simulation example in a tracking system with three-sensors is shown in Section 5 where local, distributed fusion and centralized smoothers are compared. Finally, the conclusion is drawn.

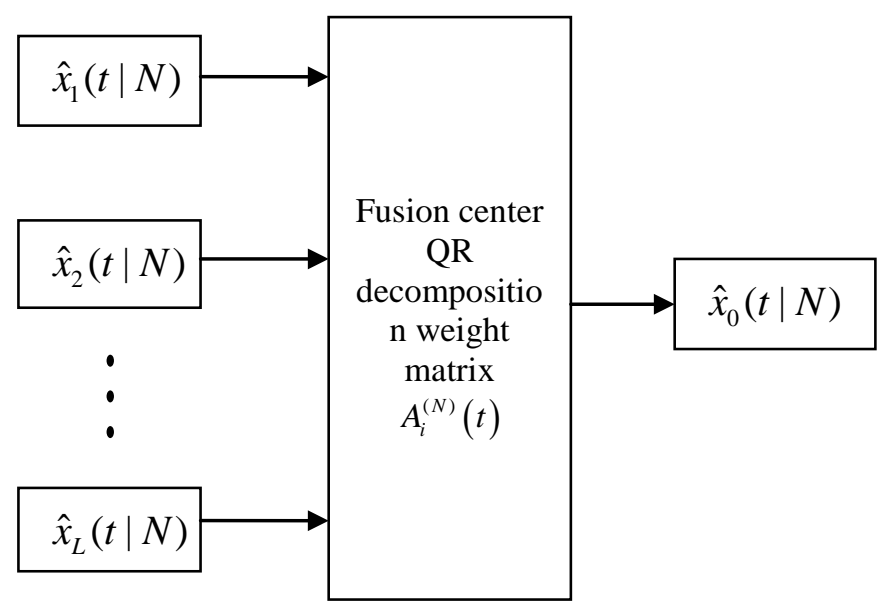

Fig.1. Distributed state fusion filtering principle

\section{Results and Analysis}

\section{A . Preliminaries}

Consider the discrete time-varying linear stochastic control system with L sensors, Target state model and measurement model are described below:

Target state model and measurement model are described below.

$$
\begin{aligned}
& x(t+1)=\Phi(t) x(t)+B(t) u(t)+\Gamma(t) w(t) \\
& y_{i}(t)=H_{i}(t) x(t)+v_{i}(t), i=1,2, \ldots, L
\end{aligned}
$$

where $x(t) \in R^{n}$ is the state, $y_{i}(t) \in R^{m i}$ is the measurement, $u(t) \in R^{p}$ is a known control input, $w(t) \in R^{r}$ and $v_{i}(t) \in R^{m i}$ are white noises, and $, \Phi(t), B(t), \Gamma(t), H_{i}(t)$, are time-varying matrices with suitable dimensions. The subscript i denotes the the sensor and is the number of sensors.
In the following, $I_{n}$ denotes the $n \times n$ identity matrix,and 0 denotes the zero matrix with suitable dimension.

$$
\begin{aligned}
& E\left(w(t) w^{T}(k)\right)=Q_{w}(t) \delta_{t k} \\
& E\left(v_{i}(t) v_{j}^{T}(k)\right)=R_{i j}(t) \delta_{t k}
\end{aligned}
$$

where $R_{i i}(t)$ is the variance of $v_{i}(t)$, i.e., $R_{i}(t)$ the symbol $\mathrm{E}$ denotes the mathematical expectation, the superscript $\mathrm{T}$ denotes the transpose, and $\delta_{t k} \mathrm{~d}$ is the Kronecker delta function.

Assumption 2. The initial state $x(0)$ is independent of $w(t)$ and $v_{i}(t), \mathrm{i}=1,2, \ldots, L$, and

$$
\begin{aligned}
& E[x(o)]=u_{0}, \\
& E\left[\left(x(0)-u_{0}\right)\left(x(0)-u_{0}\right)^{T}\right]=P_{0} .
\end{aligned}
$$

(a)The system is a known time sequences, or is a linear function about

$$
\left(y_{i}(t), y_{i}(t-1), \ldots y_{i}(1)\right), i=1,2, \ldots, L
$$

(b) Unbiasedness, namely, $E\left[\hat{x}_{0}(t \mid N)\right]=E[x(t)]$

(c) Optimality, namely, to fi nd the optimal weights $A_{i}^{(N)}(t), \mathrm{i}=1,2, \ldots, \mathrm{L}$ to minimize the traces of variance matrices of the weighted fusion smoothers $\hat{x}_{0}(t \mid N)$ respectively.

(d)the initial state of the system is not related

\section{B. QR Decompose Definition}

If $\mathrm{n} \times \mathrm{n}$ real non-singular matrix $\mathrm{A}$ can be decomposed into the orthogonal matrix $\mathrm{Q}$ and the real non-singular upper triangular matrix $\mathrm{R}$, ie $\mathrm{A}=\mathrm{QR}$, we claimed that the decomposition of the matrix $\mathrm{A}$ QR decomposition; thus $\mathrm{A}$ is $\mathrm{m}^{*} \mathrm{n}$ column matrix of full rank, if $\mathrm{A}=\mathrm{QR}$, where $\mathrm{Q}$ is the $\mathrm{m}^{*} \mathrm{n}$ matrix $\mathrm{QTQ}=\mathrm{E}(\mathrm{Q}$ is the orthogonal matrix of colum$\mathrm{ns}), \mathrm{R}$ is a non singular upper triangular matrix of the matrix A, also known as QR decomposition.

\section{Fusion process}

Based on the local smoothers and cross-covariance matrices, one has the following distributed frisiod interval smoothers in the linear minimum variance sense. with multiple sensors has the optimal weighted fusionfixed interval Kalman smoothers,

$$
\hat{x}_{0}^{\sim}(t \mid N)=A_{1}^{(N)}(t) x_{1}(t \mid N)+A_{2}^{(N)}(t) x_{2}(t \mid N)+\ldots A_{L}^{(N)}(t) x_{L}(t \mid N), N>0
$$

where local smoothers $\hat{x}_{0}(t \mid N), \mathrm{i}=1,2, \ldots, \mathrm{L}$ are computed by

$$
\hat{x}_{i}(t \mid N)=x_{i}(t \mid t-1)+\sum_{k=0}^{N-t} M_{i}(t \mid t+k) \varepsilon_{i}(t+k)
$$


where the gain matrix $M_{i}(t \mid t+k)$ is computed by

$$
\begin{aligned}
& M_{i}(t \mid t+k)=P_{i}(t \mid t-1) \Phi_{i}^{T}(t+k, t) H_{i}^{T}(t+k) Q_{\varepsilon_{i}}^{-1}(t+k) \\
& \Phi_{i}^{T}(t+k, l)=\prod_{s=l}^{t+k-1}\left[\Phi(s)-K_{P_{i}}(s) H_{i}(s)\right]^{T} \\
& \Phi_{i}(s, s)=I_{n}
\end{aligned}
$$

The optimal matrix weightes, $A_{1}^{(N)}(t)$ i $=1,2, \ldots$, L are computed by

$$
A^{(N)}(t)=\sum^{-1}(t \mid N) e\left[e^{T} \sum^{-1}(t \mid N) e\right]^{-1}
$$

Where $\sum(t \mid N)=\left(P_{i j}(t \mid N)\right)_{n l \times n l}$ is an $n l \times n l$ matrix

$A^{(N)}(t)=\left[A_{1}^{(N)}(t), A_{2}^{(N)}(t), \ldots A_{L}^{(N)}(t)\right]^{T}$ and

$e=\left[I_{n}, \ldots I_{n}\right]^{T}$ are $n l \times n l$ matrices.

The variance of the matrix weighting optimal fusion smoother is computed by

$$
P_{0}(t \mid N)=\left(e^{T} \sum^{-1}(t \mid N) e\right)^{-1}
$$

The fixed-interval smoothing error variance matrix is given by

$$
\begin{aligned}
& P_{i}(t \mid N)=P_{i}(t \mid t-1)-\sum_{k=0}^{N-t} M_{i}(t \mid t+k) Q_{\varepsilon i}(t+k) M_{i}^{T}(t \mid t+k) \\
& P_{i j}(t \mid N)=\Psi_{i}(t \mid N) P_{i j}(t \mid t-1) \Psi_{j}^{T}(t \mid N)+ \\
& \sum_{k=0}^{N-t} G_{i}(t \mid t+k) Q_{i j}(t+k) G_{j}^{T}(t \mid t+k)
\end{aligned}
$$

where covariance matrices where $P_{i j}(t \mid N)$ i, j =1,2,.., $L$ are computed by (12) and (13)..

Among: $G_{i}(t \mid t+k)=\left[G_{i}^{w}(t \mid t+k), G_{i}^{v}(t \mid t+k)\right]$ are defined by

$$
\Psi_{i}(t \mid N)=I_{n}-\sum_{k=0}^{N-t} M_{i}(t \mid t+k) H_{i}(t+k) \Phi_{i}(t+k, t),
$$

$$
\begin{aligned}
G_{i}^{w}(t \mid t+k)=- & \sum_{k=0}^{N-t} M_{i}(t \mid t+l) H_{i}(t+l) \Phi_{i}(t+l, t+k+1) \times \Gamma(t+k), \\
& k=0,1, \ldots N-t-1, G_{i}^{w}(t \mid N)=0, \\
G_{i}^{v}(t \mid t+k)= & \sum_{k=0}^{N-t} M_{i}(t \mid t+l) H_{i}(t+l) \Phi_{i}(t+l, t+k+1)
\end{aligned}
$$

$$
\times K_{P i}(t+k)-M_{i}(t \mid t+k),
$$

The application of QR decomposition in weight matrix :

QR decomposition of the covariance matrix:

$$
\left(P_{i j}(t \mid N)\right)_{n l \times n l}=Q_{i j} R_{i j}
$$

$Q_{i j}=\left(\begin{array}{ccc}q_{11} & \ldots & q_{1 j} \\ \vdots & \ddots & \vdots \\ q_{i 1} & \cdots & q_{i j}\end{array}\right)$

$R_{i j}=\left(\begin{array}{ccc}r_{11} & r_{m n} & r_{i j} \\ 0 & \ddots & \\ 0 & 0 & r_{i j}\end{array}\right)$

Yield:

$A^{(N)}(t)=Q_{i j}^{-1} R_{i j}^{-1} e\left[e^{T} Q_{i j}^{-1} R_{i j}^{-1} e\right]^{-1}$

\section{Conclusion}

Consider the tracking system with five-sensors:

$x(t+1)=\left(\begin{array}{ccc}1 & T & T^{2} / 2 \\ 0 & 1 & T \\ 0 & 0 & 1\end{array}\right) x(t)+[0 ; 0 ; 1] w(t)$

$y_{i}(t)=H_{i}(t) x(t)+v_{i}(t), i=1,2, \ldots, L$

where $\mathrm{T}$ is the sampling period, $\mathrm{i} y_{i}(t), \mathrm{i}=1,2,3$ are the measurement signals of five-sensors with the measurement matrices $\quad H(:,:, 1)=[1,0,0] \quad H(:,:, 2)=[0,1,0]$ $H(:,:, 3)=[0,0,1] H(:,:, 4)=[0,0,1] H(:,:, 5)=[1,1,0]$ respectively, the Gaussian white noise $w(t)$ with zero mean and variance $\sigma_{w}=1$, and Gaussian white noise, $\varepsilon_{i}(t) \mathrm{i}=1,2,3$ ,4,5with zero mean and varance $\sigma_{\varepsilon_{1}}=5, \sigma_{\varepsilon_{2}}=5, \sigma_{\varepsilon_{3}}=5$, $\sigma_{\varepsilon_{4}}=5, \sigma_{\varepsilon_{4}}=5$, are independent of $w(t)$. 


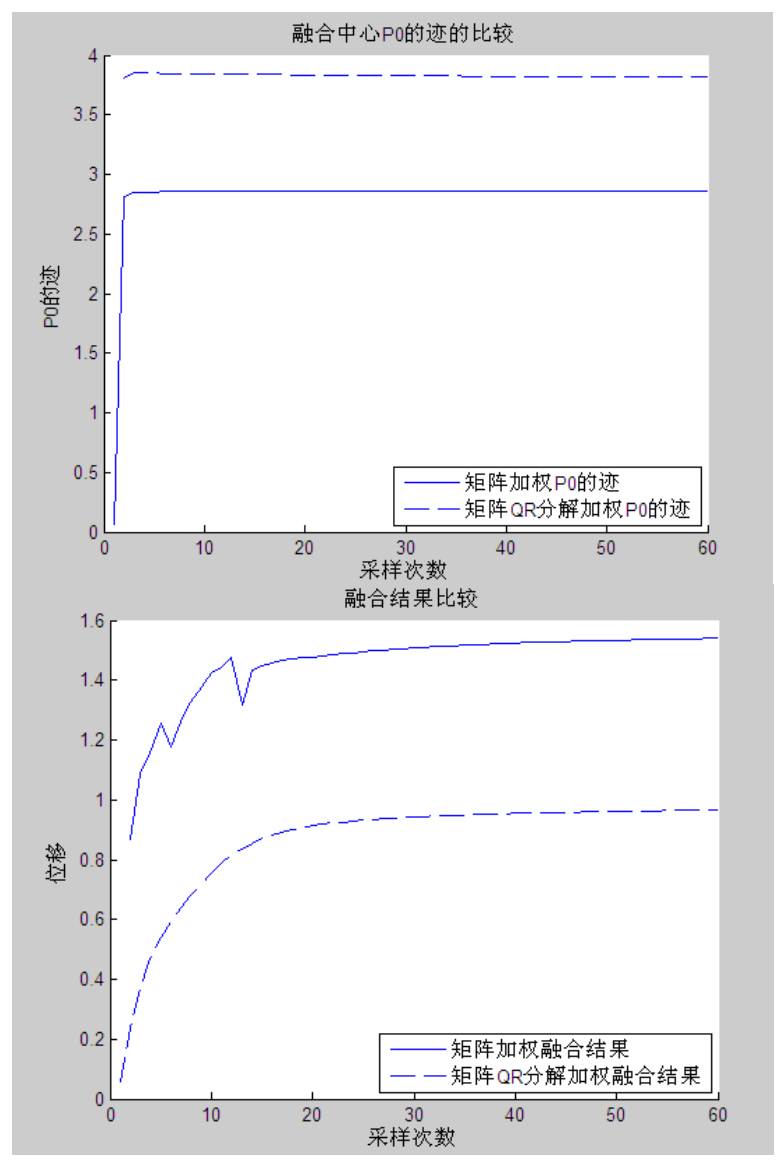

Fig. 2. Comparison of the fusion center $P_{0}$ and Comparison of fusion results

In the simulation, we take $\mathrm{T}=300, x(0)=[0 ; 0 ; 0]$ $P_{0}=0.1 *$ eye $(3) \quad \mathrm{N}=300$. $\mathrm{By}$ the above figure although $\operatorname{tr} P_{Q R}(t \mid N)<\operatorname{tr} P_{0}(t \mid N)$,QR decomposition weighted fusion filter Still changed fault tolerance of $\hat{x}_{i}(t \mid N)$ in a certain extent.

The use of the QR decomposition method, the matrix inverse calculation problem is solved to some extent, promote the weighted matrix algorithm optimization. There is a certain sense in the engineering application.

\section{Acknowledgments}

This research is partially supported by the Combination of the Ministry of education project in Guangdong Province : 2010B090400457 , 2011B090400269 , 2011A091000028, 2012B091000089 , 2012B091100383, 2012A080103010, 2011A091000046, 2012A080107007

\section{References}

[1] WIENER N. Extrapolation,Ineerpolation \& Smoothing of Sationary Time Series[M]. M.I.T.Press Cambridge,Mass.,1949

[2] N.A. Carlson, Federated square root filter for decentralized parallel processes, IEEE Transactions on Aerospace and Electronic Systems 26, 3 (May 1990), 517-525.

[3] KALMAN R E. A New Appraoch to Linear Filtering and Prediction Problem[J]. Trans. ASME Basic Eng.,1960, 82(D) :34-45.

[4] Z. L. Deng and R. E. Qi, "Multi-sensor information fusion sub-optima ls teady-state Kalman filter," Chinese Science Abslraels, vol. 6, pp. 183184, Feb. 2000.

[5] QIANG Gan , HARRIS C J . Comparison of two measurement fusion methods $\mathrm{f}$ or Kalman-filter- based multi-sensor data fusion [ J ] . I EEE Trans on Aerospace and Electronic Systems , 2001 , 37(1):273 - 280.

[6] K.H. Kim, Development of track-to-track fusion algorithm, in:Proceeding of the American Control Conference (ACC), 1994, 1037"C1041.

[7] H. Chen, T. Kirubarajan, Y. Bar-Shalom, Performance limits of track-totrack fusion versus centralized estimation: Theory and application, IEEE Transactions on Aerospace and Electronic Systems 39 (2) (2003) 386 "C398. 